\title{
Metabólitos séricos da vitamina D não se correlacionam com pressão arterial em adolescentes
}

\author{
There are no association between vitamin D \\ metabolites and blood pressure in adolescents
}

Barbara Santarosa Emo Peters', Janaína Pivetta Roque', Mauro Fisberg ${ }^{2}$, Lígia Araújo Martini'

Departamento de Nutrição Faculdade de Saúde Pública, Universidade de São Paulo (USP), São Paulo, SP, Brasil ${ }^{2}$ Centro de Atendimento e Apoio ao Adolescente Universidade Federal de São Paulo (Unifesp) São Paulo, SP, Brasil
Correspondência para: Lígia Araújo Martini Departamento de Nutrição, Faculdade de Saúde Pública, Universidade de São Paulo Av. Dr. Arnaldo, 715 - Cerqueira César

01246-904 - São Paulo, SP, Brasil Imartini@usp.br

Recebido em 7/Ago/2008 Aceito em 12/Dez/2008

\begin{abstract}
RESUMO
Objetivo: O objetivo deste estudo foi avaliar a associação entre o estado nutricional da vitamina D, a adiposidade e a pressão arterial (PA) em adolescentes. Métodos: Foi realizada avaliação antropométrica, da composição corporal, da ingestão alimentar, de medidas bioquímicas e aferição da PA de 205 adolescentes, com média de idade de 18,2 anos. Resultados: Destes, 12,19\% apresentaram PA elevada. 0 nível sérico médio da $250 \mathrm{HD}$ foi $29,2(0,8) \mathrm{ng} / \mathrm{mL}$, e $62 \%$ dos adolescentes apresentaram insuficiência de vitamina D. Não foi encontrada correlação significativa entre a PAS e a PAD com a $25 \mathrm{OHD}$ e a $1,25(\mathrm{OH})_{2} \mathrm{D}$. Houve correlação negativa entre a PAD com os níveis séricos de adiponectina, e tanto a PAS quanto a PAD apresentaram correlação positiva com a circunferência da cintura em ambos os sexos. Conclusão: Não houve relação entre os níveis séricos de vitamina $D$ e a PA. Porém, a gordura visceral apresenta risco potencial para elevação da PA em adolescentes. Arq Bras Endocrinol Metab. 2009;53(4):416-24.

Descritores

Pressão arterial; vitamina D; cálcio; adiponectina; adolescentes
\end{abstract}

\section{ABSTRACT}

Objetives: The purpose of this study was to evaluate the relationships between serum levels of vitamin D and blood pressure (BP) in adolescents. Methods: The anthropometric measurements, body composition, dietary intake, blood pressure and biochemical measurements was undertaken. Results: Two hundred and five adolescents, 106 boys and 99 girls, mean aged 18.2 years old, participate of the study. Elevated BP was observed in $12.19 \%$ of the adolescents. Mean 250HD concentration was $29.2(0.8) \mathrm{ng} / \mathrm{mL}$. Vitamin D insufficiency was observed in $62 \%$ of adolescents. There were no correlation between systolic and diastolic BP with $25 \mathrm{OHD}$ and $1,25(\mathrm{OH})_{2} \mathrm{D}$ concentration. An inverse correlation between adiponectin and diastolic BP was observed and positive significant correlation was observed between waist circumference with systolic and diastolic BP in both boys and in girls. In conclusion, no relationships between vitamin D levels and blood pressure were observed. Conclusion: However, the intra-abdominal adiposity offers potential risk to BP elevation in adolescents. Arq Bras Endocrinol Metab. 2009;53(4):416-24.

\section{Keywords}

Blood pressure; vitamin D; calcium; adiponectin; adolescents

\section{INTRODUÇÃO}

A portância da vitamina D para a saúde óssea é bem estabelecida. Entretanto, estudos recentes têm demonstrado que a deficiência dessa vitamina pode estar associada ao diabetes, a doenças autoimunes, a diversos tipos de câncer e, também, com a hipertensão (1-4).
O mecanismo provável está relacionado com a inibição do sistema renina-angiotensina (5-8). A renina é uma enzima que catalisa a divisão da angiotensina I do angiotensinogênio produzido no fígado. A enzima conversora de angiotensina (ECA) catalisa a clivagem de angiotensina I para a forma angiotensina II, um peptídeo 
que pode aumentar a pressão sanguínea pela indução da constrição de pequenas artérias e aumento da retenção de sódio e água pelo organismo. A taxa de síntese de angiotensina II é dependente da renina (8). Os camundongos knockout para o receptor da vitamina D (VDR) apresentam níveis elevados de renina e também da pressão arterial (PA). Além disso, a administração da $1,25(\mathrm{OH})_{2} \mathrm{D}$ em camundongos wild type diminui a expressão do gene da renina $(6,9)$. Dessa forma, a ativação inapropriada do sistema renina-angiotensina está interligada ao desenvolvimento da hipertensão, e adequados níveis de vitamina $\mathrm{D}$ podem ser importantes para a diminuição do risco da elevação na pressão sanguínea.

De fato, estudos epidemiológicos recentes têm demonstrado que altos níveis séricos da 25-hidroxivitamina $\mathrm{D}(25 \mathrm{OHD})$ estão associados com menor pressão arterial média (PAM) e com redução da prevalência de hipertensão (10-13). Por outro lado, a elevação na PA está associada com a gordura visceral (13), assim como níveis séricos de vitamina $\mathrm{D}$ também se apresentam reduzidos em indivíduos com maior quantidade de gordura corporal (14-16).

Entretanto, esses estudos foram realizados em adultos e idosos e até o presente momento não há pesquisas envolvendo esse tema em adolescentes. Assim sendo, o objetivo deste estudo foi avaliar a associação entre os níveis séricos da vitamina $\mathrm{D}$ com a PA e a adiposidade em adolescentes.

\section{MÉTODOS}

\section{Amostra}

O estudo foi realizado em uma escola pública da cidade de Indaiatuba, interior de São Paulo, durante abril e maio de 2006. Inicialmente, foram selecionados 330 adolescentes regularmente matriculados nos cursos do período matutino, vespertino e noturno, com idade entre 16 e 20 anos. Foram adotados como critério de exclusão: presença de doenças renais, cardiovasculares, hepática, diabetes, uso de corticosteroides e anti-inflamatórios, e estar gestante/lactante. Concordaram em participar deste estudo 205 estudantes (106 meninos e 99 meninas). Todos os participantes ou seus responsáveis assinaram o termo de consentimento, aprovado pelo Comitê de Ética em Pesquisa da Faculdade de Saúde Pública da Universidade de São Paulo, de acordo com os requisitos da Resolução CNS n ${ }^{0}$ 196/1996 e o protocolo de pesquisa $n^{0} 1.316$.
Foram realizadas avaliações antropométrica, da composição corporal e da ingestão alimentar e bioquímica da amostra. Alguns adolescentes não completaram a avaliação da ingestão alimentar e não concordaram com a coleta de amostras de sangue, assim a avaliação da ingestão alimentar foi realizada em 163 adolescentes e, destes, 132 realizaram análises bioquímicas.

\section{Antropometria e composição corporal}

Para avaliação antropométrica foram aferidos o peso, a altura e as circunferências do braço (CB), cintura (CC) e quadril (CQ). Os adolescentes foram pesados em uma balança mecânica para adultos com escala antropométrica, capacidade $150 \mathrm{~kg}$, e fração de $100 \mathrm{~g}$ da marca Welmy ${ }^{\circledR}$, e para medir a estatura foi utilizada a barra metálica acoplada à balança. O índice de massa corporal (IMC) foi calculado dividindo-se o peso em quilos $(\mathrm{kg})$ pelo quadrado da altura em metros, e os adolescentes foram classificados conforme os critérios propostos pela Organização Mundial de Saúde de 1995, a partir das curvas do National Center for Health Statistics (17). Para a aferição das CB, CC e CQ, utilizou-se uma fita métrica, flexível e inextensível. A CB foi realizada no ponto médio entre a borda súpero-lateral do acrômio e o olecrano, a CC no ponto médio entre o último arco costal e a crista ilíaca do indivíduo em pé, com a leitura feita no momento da expiração, e a CQ foi realizada na região de maior perímetro entre a cintura e a coxa do indivíduo.

Para a avaliação da composição corporal, a impedância bioelétrica foi realizada com o aparelho tetrapolar Quantum BIA - 101Q da marca RJL-101 (Detroit, MI, Estados Unidos). Para classificar os adolescentes quanto à porcentagem de gordura corporal, foram adotados como valores normais aqueles sendo $<30 \%$ para meninas e $<20 \%$ para meninos, preconizado, em 2004, por Rodriguez e cols. (18).

\section{Ingestão alimentar}

Foi utilizado o registro alimentar de três dias para avaliar a ingestão média de energia, proteína, cálcio, vitamina $\mathrm{D}$, potássio, sódio e álcool. Os estudantes foram questionados quanto ao uso ou não de suplementos de cálcio e vitamina $\mathrm{D}$. Os dados de ingestão alimentar foram analisados utilizando-se o software Nutrition Data System for Research Grad-Pack (2005) desenvolvido pelo Nutrition Coordinating Center (NCC), Universidade de Minnesota, Mineápolis, MN. A avaliação da 
adequação de ingestão dos micronutrientes foi realizada seguindo as recomendações das Dietary Reference Intakes (DRI) para esse grupo etário. Quanto à ingestão de álcool, foram utilizadas as recomendações para adultos das V Diretrizes Brasileiras de Hipertensão Arterial, nas quais o recomendado para homens é de até $30 \mathrm{~mL} /$ dia e para mulheres, até $15 \mathrm{~mL} /$ dia.

\section{Pressão arterial}

As medidas da PA foram realizadas na própria escola por enfermeiros devidamente treinados e capacitados, de acordo com as recomendações das V Diretrizes Brasileiras de Hipertensão Arterial (19). Antes da aferição, os adolescentes permaneceram em repouso por, pelo menos, 5 minutos em ambiente tranquilo. Foram realizadas três medidas da PA, com intervalo de 1 minuto entre elas, sendo a média das duas últimas considerada a PA do indivíduo. Durante a aferição, os adolescentes permaneceram na posição sentada. Foram utilizados os seguintes equipamentos: esfigmomanômetros de coluna de mercúrio, devidamente testados e calibrados, e estetoscópios duplos e manguitos de larguras correspondentes a $40 \%$ da circunferência do braço utilizado.

Para a classificação da PA dos adolescentes abaixo dos 18 anos de idade, foram considerados os valores abaixo do percentil 90 como normotensão, desde que inferiores a $120 \times 80 \mathrm{mmHg}$; entre os percentis 90 e 95 como limítrofe ("pré-hipertensão", de acordo com o The Fourth Report on the Diagnosis, Evaluation, and Treatment of High Blood Pressure in Children and Adolescents); entre os percentis 95 e 99 mais $5 \mathrm{mmHg}$ como hipertensão estágio 1 , e superior ao percentil 99 mais $5 \mathrm{mmHg}$ como hipertensão estágio 2 , salientando-se que qualquer valor igual ou superior a 120 x 80 $\mathrm{mmHg}$ em adolescentes, mesmo que inferior ao percentil 95, foi considerado limítrofe. Para os adolescentes acima de 18 anos, considerou-se a seguinte classificação: PA sistólica (PAS) < $120 \mathrm{mmHg}$ e PA diastólica $(\mathrm{PAD})<80 \mathrm{mmHg}$ como ótima; PAS < $130 \mathrm{mmHg}$ e PAD < $85 \mathrm{mmHg}$ como normal; PAS entre 130 e 139 mmHg e PAD entre 85 e 89 mmHg como limítrofe; PAS entre 140 e $159 \mathrm{mmHg}$ e PAD entre 90 e $99 \mathrm{mmHg}$ como hipertensão estágio 1; PAS entre 160 e $179 \mathrm{mmHg}$ e PAD entre 100 e $109 \mathrm{mmHg}$ como hipertensão estágio 2; PAS $\geq 180 \mathrm{mmHg}$ e $\mathrm{PAD} \geq 110$ $\mathrm{mmHg}$ como hipertensão estágio 3. Quando a PAS e a PAD de um dos adolescentes situaram-se em categorias diferentes, a maior foi utilizada para classificação da PA.
Para a análise estatística, foram considerados valores normais de PA aqueles classificados como ótimos, normais e limítrofes, e foram considerados valores de PA elevados aqueles classificados como hipertensão.

\section{Atividade física}

Todos os adolescentes completaram um questionário de atividade física, desenvolvido e validado para adolescentes, em 2006, por Florindo e cols. (20). Esse questionário apresenta 17 questões divididas em dois blocos: 1) esportes ou exercícios físicos (15 questões) e 2) atividades físicas de locomoção para a escola (duas questões). Somando os blocos 1 e 2 , o questionário apresenta o escore semanal de atividade física praticada pelos adolescentes. Para o cálculo do nível de atividade física, foi utilizado o resultado final do escore como variável contínua, e como variável dicotômica foi utilizado o ponto de corte de $\geq 300 \mathrm{~min} / \mathrm{sem}$ como atividade física moderada ou vigorosa e $<300 \mathrm{~min} / \mathrm{sem}$ como atividade física leve ou sedentária (2l).

\section{Exposição solar}

Quanto à exposição solar, os adolescentes foram questionados em relação ao uso diário de protetor solar (sim ou não) e se praticavam atividade física expostos ao sol (sim ou não).

\section{Análises laboratoriais}

As amostras de sangue foram coletadas por enfermeiros treinados e capacitados, com os adolescentes em jejum de 12 horas. Após a coleta, as amostras foram centrifugadas a $2.000 \mathrm{rpm}$ por $10 \mathrm{~min}$ em temperatura ambiente. Os soros foram armazenados no freezer $-80^{\circ} \mathrm{C}$ para posterior análise do cálcio sérico total, do paratormônio intacto (PTHi), da $25 \mathrm{OHD}$, da $1,25(\mathrm{OH})_{2} \mathrm{D}$ e da adiponectina. $\mathrm{O}$ cálcio sérico total foi analisado pelo método colorimétrico por O-cresolftaleína; valores normais: 8,6 a $10,2 \mathrm{mg} / \mathrm{dL}$. O PTHi foi avaliado por eletroquimioluminescência (Nichols Institute, San Clemente CA); valores normais: 15 a $65 \mathrm{pg} / \mathrm{mL}$. Os níveis séricos da 25OHD foram avaliados por kit de radioimunoensaio (DiaSorin, Stillwater, MN); com coeficiente de variação $(\mathrm{CV})$ intraensaio de $8,6 \%$ a $12,5 \%$ e CV interensaio de $8,2 \%$ a $11,0 \%$. Os níveis séricos da $1,25(\mathrm{OH})_{2} \mathrm{D}$ foram avaliados por kit de radioimunoensaio (DiaSorin, Stillwater, MN); com CV intraensaio de $6,8 \%$ a $11,3 \%$ e CV interensaio de $11,1 \%$ a $14,6 \%$. $\mathrm{A}$ adiponectina foi avaliada por ELISA (ALPCO Diag- 
nosis, Salem, NH); com CV intraensaio de 3,08\% a $4,84 \%$ e CV interensaio de $1,76 \%$ a $5,05 \%$.

\section{Pontos de corte da vitamina D}

Os pontos de corte utilizados para classificar a deficiência e a insuficiência de vitamina $\mathrm{D}$ foram os propostos, em 2006, por Bischoff-Ferrari e cols. (22), que define a deficiência de vitamina $\mathrm{D}$ quando níveis séricos da $25 \mathrm{OHD}$ são $\leq 10 \mathrm{ng} / \mathrm{mL}$ e insuficiência de vitamina $\mathrm{D}$ quando os níveis séricos da $25 \mathrm{OHD}$ estão entre 10 e $30 \mathrm{ng} / \mathrm{mL}$.

\section{Análise estatística}

Para a análise estatística, realizou-se o teste Kolmogorov-Smirnov, para avaliar a aderência das variáveis à distribuição normal. Para examinar a relação entre as variáveis categóricas, foi utilizado o teste qui-quadrado ou, quando necessário, o teste exato de Fisher (presença de valores esperados menores que 5). Para analisar a relação da PAS e da PAD com as demais variáveis, foi utilizado o coeficiente de correlação de Spearman, em virtude da ausência de distribuição normal dessas variáveis. As comparações anteriores foram refeitas, ajustando-se para os valores de IMC por meio da análise de covariância simples (Oneway Ancova) e do coeficiente de correlação parcializada de Spearman. As análises estatísticas foram realizadas com o auxílio dos programas estatísticos SPSS for Windows, versão 11.0 (SPSS Inc., Chicago, IL, Estados Unidos), e SAS System for Windows (statistical analysis system), versão 8.02 (SAS Insti- tute Inc, Cary, NC, Estados Unidos). Foi considerado grau de significância de $5 \%$. Os resultados serão apresentados na forma de média e erro-padrão.

Para controlar o efeito do consumo de energia nos micronutrientes avaliados, foi aplicado o método do nutriente residual, proposto, em 1998, por Willett e Stamper (23).

\section{RESULTADOS}

As características gerais dos adolescentes estudados estão apresentadas na tabela 1 . Não houve diferença estatisticamente significativa entre os gêneros em relação ao IMC. Apenas 1,0\% dos adolescentes apresentou baixo peso, $82,9 \%$ eram eutróficos, $11,7 \%$ apresentaram sobrepeso e $4,4 \%$ eram obesos. Como esperado, a porcentagem de gordura corporal foi significantemente maior nas meninas do que nos meninos $[31,51(0,80) \%$ versus $15,23(0,47) \%$ respectivamente; $\mathrm{p}<0,001$ ], porquanto $16,0 \%$ dos meninos e $50,5 \%$ das meninas apresentaram excesso de gordura corporal.

Apresentaram níveis de PA elevados 12,19\% dos adolescentes. Tanto a PAS quanto a PAD foram significativamente mais elevadas nos meninos do que nas meninas [PAS: 121,27 $(1,07) \mathrm{mmHg}$ versus $110,61(1,0) \mathrm{mmHg}$, respectivamente, $\mathrm{p}<0,001$; $\mathrm{PAD}: 77,36(0,98) \mathrm{mmHg}$ versus $72,53(0,86) \mathrm{mmHg}$, respectivamente, $\mathrm{p}<0,001]$.

Os meninos apresentaram, em média, maior escore de atividade física em minutos por semana comparado às meninas $[554,10(40,7) \mathrm{min} / \mathrm{sem}$ versus 324,85 $(27,7) \mathrm{min} / \mathrm{sem}$, respectivamente, $\mathrm{p}<0,001]$. Além

\begin{tabular}{|c|c|c|c|c|}
\hline & $\begin{array}{c}\text { Total } \\
\mathrm{n}=205\end{array}$ & $\begin{array}{c}\text { Meninos } \\
n=106\end{array}$ & $\begin{array}{c}\text { Meninas } \\
\mathrm{n}=99\end{array}$ & $\mathbf{p}$ \\
\hline Idade (anos) & $18,25(0,07)$ & $18,09(0,09)$ & $18,42(0,1)$ & 0,011 \\
\hline Peso (kg) & $64,61(0,89)$ & $69,42(1,17)$ & $59,46(1,15)$ & 0,000 \\
\hline Altura (m) & $1,68(0,01)$ & $1,75(0,01)$ & $1,61(0,01)$ & 0,000 \\
\hline IMC $\left(\mathrm{kg} / \mathrm{m}^{2}\right)$ & $22,81(0,28)$ & $22,79(0,37)$ & $22,82(0,42)$ & 0,956 \\
\hline Gordura corporal (\%) & $23,09(0,73)$ & $15,23(0,47)$ & $31,51(0,80)$ & 0,000 \\
\hline $\mathrm{CC}(\mathrm{cm})$ & $75,40(0,72)$ & $77,93(1,01)$ & $72,69(0,95)$ & 0,000 \\
\hline $\mathrm{CB}(\mathrm{cm})$ & $27,09(0,27)$ & $28,27(0,39)$ & $25,83(0,32)$ & 0,000 \\
\hline $\mathrm{CQ}(\mathrm{cm})$ & $97,43(0,54)$ & $97,75(0,75)$ & $97,09(0,77)$ & 0,000 \\
\hline PAS (mmHg) & $116,12(0,82)$ & $121,27(1,07)$ & $110,61(1,0)$ & 0,000 \\
\hline $\mathrm{PAD}(\mathrm{mmHg})$ & $75,02(0,68)$ & $77,36(0,98)$ & $72,53(0,86)$ & 0,000 \\
\hline $\mathrm{AF}(\mathrm{min} / \mathrm{sem})$ & $443,39(26,1)$ & $554,10(40,7)$ & $324,85(26,7)$ & 0,000 \\
\hline
\end{tabular}

IMC: índice de massa corporal; CC: circunferência da cintura; CB: circunferência do braço; CQ: circunferência do quadril; PAS: pressão arterial sistólica; PAD: pressão arterial diastólica; AF: atividade física. 
disso, $70,8 \%$ dos meninos e $29,2 \%$ das meninas afirmaram praticar atividade física moderada ou intensa $(\mathrm{p}=0,002)$, enquanto apenas $6,8 \%$ dos adolescentes relataram não praticar nenhum esporte ou exercício físico e nenhuma atividade física de locomoção para a escola.

Nenhum dos adolescentes declarou fazer uso de suplementos de cálcio ou de vitamina $\mathrm{D}$. A ingestão média de cálcio, vitamina $\mathrm{D}$, potássio e sódio entre os adolescentes foi de $738,72(34,3) \mathrm{mg} / \mathrm{dia}, 3,36(0,2)$ $\mu \mathrm{g} / \mathrm{dia}, 2.521,74(80,17) \mathrm{g} /$ dia e $3.578,05(106,4)$ $\mathrm{g} /$ dia respectivamente, sendo a ingestão dos meninos estatisticamente maior do que das meninas (Tabela 2 ). Quanto ao consumo de cálcio, 6,7\% dos adolescentes apresentaram ingestão próxima ao valor recomendado $(1.300 \mathrm{mg} /$ dia para adolescentes < 19 anos e $1.000 \mathrm{mg} /$ dia para adolescentes $>19$ anos). Quanto à vitamina $\mathrm{D}, 14,9 \%$ dos adolescentes atingiram a recomendação de vitamina $\mathrm{D}$ para adolescentes $(5,0$ $\mu \mathrm{g} / \mathrm{dia})$. Apenas 3,1\% dos adolescentes consumiram o recomendado para o potássio $(4,7 \mathrm{~g} / \mathrm{dia})$. Quanto ao consumo de sódio, $84,0 \%$ dos adolescentes ultrapassaram a UL (upper limit) para esse micronutriente $(>2,2 \mathrm{~g} / \mathrm{dia})$.

Em relação à ingestão de álcool, 7,4\% dos meninos e $1,2 \%$ das meninas ultrapassaram o consumo recomendado para adultos.
Pode-se notar na tabela 3 que a média do nível sérico da 25OHD nos adolescentes foi de 29,2 $(0,8) \mathrm{ng} /$ $\mathrm{mL}$. Nenhum estudante apresentou deficiência de vitamina $\mathrm{D}(<10 \mathrm{ng} / \mathrm{mL})$. Entretanto, 63,9\% dos meninos e $60,6 \%$ das meninas apresentaram insuficiência de vitamina D (10 a $30 \mathrm{ng} / \mathrm{mL})$.

Não houve diferença estatisticamente significativa nos níveis séricos da $25 \mathrm{OHD}$ entre os adolescentes que usavam e os que não usavam protetor solar diariamente $[29,2$ $(0,9) \mathrm{ng} / \mathrm{mL}$ versus $28,0(1,2) \mathrm{ng} / \mathrm{mL}$, respectivamente, $\mathrm{p}=0,532]$, e entre os que praticavam e os que não praticavam exercício físico expostos ao sol $[28,8(1,0) \mathrm{ng} / \mathrm{mL}$ versus $29,5(0,9) \mathrm{ng} / \mathrm{mL}$, respectivamente, $\mathrm{p}=0,676]$.

Como demonstrado na tabela 4 , houve correlação positiva e significativa entre a PAS e a PAD com o IMC, a $\mathrm{CB}$, a CC e a CQ. Houve correlação positiva entre a porcentagem de gordura corporal (\%GC) com a PAS e a PAD, tanto para os meninos quanto para as meninas. Ajustando-se os valores de PA (PAS e PAD) para o IMC, em relação à ingestão alimentar, a PAS apenas se correlacionou positivamente com a ingestão de sódio para ambos os sexos, e a PAD apresentou correlação estatisticamente significativa com a ingestão de vitamina D também para ambos os sexos.

Não foi encontrada correlação estatisticamente significativa entre a PAS e a PAD com os níveis séricos da 25OHD, da $1,25(\mathrm{OH})_{2} \mathrm{D}$ e do PTHi, e também entre

Tabela 2. Ingestão média de nutrientes dos adolescentes avaliados

\begin{tabular}{lcccc}
\hline Ingestão alimentar & $\begin{array}{c}\text { Total } \\
\mathbf{n = 1 6 3}\end{array}$ & $\begin{array}{c}\text { Meninos } \\
\mathbf{n = 8 1}\end{array}$ & $\begin{array}{c}\text { Meninas } \\
\mathbf{n = 8 2}\end{array}$ & p \\
\hline Energia (kcal/dia) & $2376,1(67,1)$ & $2687,7(108,6)$ & $2068,23(63,3)$ & 0,000 \\
Proteína (g/dia) & $87,8(2,7)$ & $100,1(4,4)$ & $75,7(2,5)$ & 0,000 \\
Cálcio (mg/dia) & $738,4(34,3)$ & $839,7(60,8)$ & $638,4(28,8)$ & 0,019 \\
Vitamina D (ug/dia) & $3,4(0,2)$ & $3,8(0,3)$ & $2,9(0,2)$ & 0,035 \\
Sódio (mg/dia) & $3578,1(106,4)$ & $4032,2(470,0)$ & $3129,5(108,7)$ & 0,000 \\
Potássio (mg/dia) & $2521,7(80,2)$ & $2857,5(129,7)$ & $2190,1(80,1)$ & 0,000 \\
Álcool (ml/dia) & $4,25(1,7)$ & $7,56(3,4)$ & $0,97(0,7)$ & 0,494 \\
\hline
\end{tabular}

Tabela 3. Valores médios dos marcadores bioquímicos avaliados

\begin{tabular}{lcccc}
\hline Marcadores bioquímicos & $\begin{array}{c}\text { Total } \\
\mathbf{n = 1 3 2}\end{array}$ & $\begin{array}{c}\text { Meninos } \\
\mathbf{n = 6 1}\end{array}$ & $\begin{array}{c}\text { Meninas } \\
\mathbf{n = 7 1}\end{array}$ & $\mathbf{p}$ \\
\hline Cálcio $(\mathrm{mg} / \mathrm{mL})$ & $9,5(0,04)$ & $9,7(0,05)$ & $9,4(0,05)$ & 0,000 \\
25(OH)D $(\mathrm{ng} / \mathrm{mL})$ & $29,2(0,77)$ & $28,7(1,09)$ & $29,6(1,08)$ & 0,558 \\
$1,25(\mathrm{OH}) 2 \mathrm{D}(\mathrm{pg} / \mathrm{mL})$ & $71,6(2,29)$ & $68,2(3,08)$ & $74,1(3,28)$ & 0,206 \\
PTHi $(\mathrm{pg} / \mathrm{mL})$ & $25,9(0,84)$ & $29,0(1,31)$ & $23,2(0,99)$ & 0,000 \\
Adiponectina $(\mu \mathrm{g} / \mathrm{mL})$ & $31,0(1,25)$ & $28,9(1,27)$ & $32,1(1,78)$ & 0,222 \\
\hline
\end{tabular}


Tabela 4. Correlações entre as pressões arteriais sistólica e diastólica com os dados de composição corporal, ingestão alimentar e marcadores bioquímicos

\begin{tabular}{|c|c|c|c|c|c|c|}
\hline & \multicolumn{3}{|c|}{ Pressão arterial sistólica $(\mathrm{mmHg})$} & \multicolumn{3}{|c|}{ Pressão arterial diastólica (mmHg) } \\
\hline & Meninos & Meninas & Total & Meninos & Meninas & Total \\
\hline \multicolumn{7}{|l|}{ Composição corporal } \\
\hline IMC (kg/m²) & $0,379^{* *}$ & $0,387^{\star \star}$ & $0,351^{* *}$ & $0,238^{*}$ & $0,246^{*}$ & $0,242^{\star \star}$ \\
\hline $\mathrm{CB}(\mathrm{cm})$ & $0,306^{\star *}$ & $0,320^{\star *}$ & $0,442^{* *}$ & 0,174 & $0,235^{\star}$ & $0,265^{\star \star}$ \\
\hline CC (cm) & $0,430^{* *}$ & $0,374^{\star \star}$ & $0,500^{* *}$ & $0,240^{*}$ & $0,200^{*}$ & $0,283^{\star \star}$ \\
\hline$C Q(\mathrm{~cm})$ & $0,230^{*}$ & $0,368^{\star \star}$ & $0,302^{* *}$ & 0,096 & $0,244^{*}$ & $0,179^{\star \star}$ \\
\hline$\% G C$ & $0,339^{* *}$ & $0,355^{\star \star}$ & $-0,215$ & $0,222^{*}$ & $0,242^{*}$ & $-0,086$ \\
\hline \multicolumn{7}{|l|}{ Nutrientes } \\
\hline Cálcio (mg/dia) & 0,114 & $-0,055$ & 0,130 & 0,074 & 0,033 & 0,115 \\
\hline Vitamina D ( $\mu \mathrm{g} / \mathrm{dia})$ & 0,096 & $-0,059$ & 0,119 & 0,157 & 0,123 & $0,191^{*}$ \\
\hline Potássio (g/dia) & 0,084 & $-0,222^{\star}$ & 0,130 & 0,117 & $-0,075$ & 0,141 \\
\hline Sódio (g/dia) & 0,142 & $-0,135$ & $0,194^{*}$ & 0,055 & $-0,053$ & 0,111 \\
\hline Álcool (mL/dia) & 0,067 & $-0,112$ & 0,022 & 0,164 & 0,100 & 0,138 \\
\hline \multicolumn{7}{|c|}{ Marcadores bioquímicos } \\
\hline Cálcio (mg/mL) & 0,016 & $-0,070$ & $0,176^{\star}$ & $-0,087$ & $-0,063$ & 0,074 \\
\hline 25(OH)D (ng/mL) & 0,026 & $-0,019$ & $-0,016$ & $-0,147$ & $-0,010$ & $-0,076$ \\
\hline $1,25(\mathrm{OH}) 2 \mathrm{D}(\mathrm{pg} / \mathrm{mL})$ & 0,165 & $-0,240$ & $-0,100$ & 0,058 & $-0,067$ & $-0,029$ \\
\hline PTHi (pg/mL) & $-0,199$ & 0,045 & 0,070 & $-0,133$ & 0,142 & 0,081 \\
\hline Adiponectina ( $\mu \mathrm{g} / \mathrm{mL})$ & $-0,250$ & $-0,117$ & $-0,251$ & $-0,334$ & $-0,260$ & $-0,333^{*}$ \\
\hline
\end{tabular}

${ }^{*} \mathrm{P}<0,05$; ${ }^{* *} \mathrm{P}<0,01$; IMC: índice de massa corporal; CB: circunferência do braço; CC: circunferência da cintura; $\mathrm{CQ}$ : circunferência do quadril; \%GC: porcentagem de gordura corporal.

parâmetros do metabolismo ósseo e da ingestão de vitamina D com metabólitos da vitamina D. Entretanto, foi encontrada correlação negativa entre a PAD com os níveis séricos de adiponectina $(\mathrm{r}=-0,333 ; \mathrm{p}=0,024)$.

\section{DISCUSSÃO}

A prevalência de hipertensão arterial no Brasil ( $\geq 140 / 90 \mathrm{mmHg}$ ) varia de $22,3 \%$ a $43,9 \%$ segundo as V Diretrizes Brasileiras de Hipertensão Arterial de 2006. Em crianças e adolescentes, a prevalência de hipertensão arterial tem sido observada entre 2\% e $13 \%$ (24). No presente estudo, 12,19\% dos adolescentes apresentaram valores de PA acima do percentil $95 \mathrm{ou}$ valor igual ou superior a $120 / 80 \mathrm{mmHg}$.

Em adolescentes, em 2007, Silva e Farias Júnior (25), ao avaliarem fatores de risco associados à PA elevada em 674 meninos e meninas de escolas públicas e privadas, da cidade de João Pessoa, PB, encontraram prevalência de 7,4\% de PA elevada (acima do percentil 95). Esses autores não encontraram correlações significativas entre os níveis de atividade física, os hábitos alimentares e o consumo de bebidas alcoólicas com a PA nestes adolescentes, apenas o excesso de peso corporal se apresentou como fator de risco potencial para elevação da PA nos adolescentes de ambos os sexos. Dados semelhantes em adolescentes foram também observados por diversos autores (26-28) e no presente estudo, no qual também não foram encontradas associações entre os níveis de atividade física e a ingestão de álcool com os valores de PAS e PAD.

Entretanto, foram encontradas correlações positivas entre a PAS e a PAD com o IMC e os parâmetros antropométricos, como $\mathrm{CB}, \mathrm{CC}$ e $\mathrm{CQ}$, sendo os valores dessas variáveis estatisticamente mais elevados nos adolescentes com PA elevada. Esses resultados reforçam achados de diversos estudos que relataram grande impacto do excesso de peso corporal sobre os níveis de PA em crianças e adolescentes $(26,27,29-33)$.

$\mathrm{O}$ excesso de gordura visceral é outro fator que também exerce grande impacto na elevação da PA. No presente estudo, além da correlação positiva entre PAS e PAD com a CC, também houve correlação positiva entre a PAS e PAD com a \%GC, tanto nos meninos quanto nas meninas. Com o objetivo de investigar a associação entre a gordura intra-abdominal (GIA) e os componentes individuais da síndrome metabólica em 324 adolescentes, um estudo transversal realizado, em 
2008, por Syme e cols. (14) verificou que os meninos com excesso de GIA apresentaram PAS estatisticamente mais elevada do que os meninos com baixa GIA.

Corroborando com a questão da gordura visceral, no presente estudo foi encontrada correlação negativa da adiponectina com a PAD. Um estudo realizado, em 2004, por Weiss e cols. (34), tendo como objetivo avaliar a relação entre obesidade e síndrome metabólica em crianças e adolescentes, encontrou correlação negativa da adiponectina com a obesidade. A adiponectina tem sido considerada determinante da gordura visceral, uma vez que este hormônio apresenta associação inversa com o volume de gordura visceral em diversos estudos (35-37). Assim, os dados encontrados neste estudo enfatizam a importância da avaliação dos compartimentos corporais para a adequada avaliação de fatores de risco ao desenvolvimento da hipertensão.

Em relação à ingestão alimentar, foi encontrada correlação positiva entre a ingestão de sódio e a PAS nos adolescentes avaliados. Estudos randomizados comparando dieta hipossódica com a dieta habitual, com ou sem redução de peso, demonstram efeito favorável, embora modesto, na redução da PA com a restrição de sal $(38,39)$. As DRIs recomendam que a ingestão de sódio não ultrapasse os 2,2 $\mathrm{g} / \mathrm{dia}$, e $84,0 \%$ dos adolescentes deste estudo ingeriam quantidade de sódio acima desse valor.

Deve-se considerar, ainda, que a ingestão de sódio neste estudo pode estar subestimada, pois não foi mensurado o sal de adição da dieta (sal dos saleiros, temperos prontos). Foi considerado apenas o sal utilizado no preparo de alimentos, assim como o sódio intrínseco e o sódio dos alimentos industrializados. De qualquer maneira, os dados encontrados no estudo sugerem que a diminuição na quantidade de sal pode trazer efeitos benéficos na diminuição da PA durante a adolescência.

$A$ ingestão de vitamina $D$ também parece exercer efeito na PA. Dados de um estudo transversal realizado, em 1985, por Sowers, Wallace e Lemke (40) sugeriram associação entre baixa ingestão de vitamina $\mathrm{D}$ (< $10 \mu \mathrm{g} /$ dia) e elevação da PA. Um estudo de intervenção, ao avaliar a deficiência de vitamina $\mathrm{D}$ em mulheres idosas, verificou que a suplementação de cálcio combinada com vitamina D (1.200 mg de cálcio + 20 $\mu \mathrm{g}$ de vitamina D por dia) apresentava melhor efeito na diminuição da PA do que apenas a suplementação de cálcio (1.200 mg/dia) (4l). Dos adolescentes avaliados neste estudo nenhum fazia uso de suplemento de vitamina $\mathrm{D}$, e apenas $14,9 \%$ dos adolescentes consumiam o recomendado pelas DRIs para a vitamina D $(5,0$ $\mu \mathrm{g} /$ dia). Possivelmente, pelo fato de a ingestão deste nutriente ter sido em média bastante reduzida [3,36 $(0,2) \mu \mathrm{g} /$ dia] nesta população, não foram encontradas associações dessa vitamina com a PA. Adicionalmente, não foi encontrada correlação entre ingestão e níveis séricos de vitamina $\mathrm{D}$, pois se estima que de $5 \%$ a $10 \%$ da vitamina $\mathrm{D}$ biodisponível seja oriunda da ingestão de alimentos, sendo a maior proporção adquirida pela síntese cutânea (42).

Em relação aos dados bioquímicos, em 2007, Snijder e cols. (43), ao avaliarem a relação da vitamina D sérica e do PTHi com a PA em 1.205 participantes do Longitudinal Aging Study Amsterdam, não encontraram associação significativa entre os níveis séricos da 25OHD com a PAS e a PAD. Entretanto, os níveis séricos de PTHi apresentaram associação significativa com a PA, tanto sistólica quanto diastólica. Ao procurar explicar esses achados, os autores discutem que nos estudos em que os níveis séricos da 25OHD apresentaram associação significativa com a $\mathrm{PA}$, a maioria da amostra apresentava níveis séricos de vitamina $\mathrm{D}$ menores que $20 \mathrm{ng} / \mathrm{mL}$. Os autores ainda salientam que essa associação não foi significativa, e apenas $10,5 \%$ da população apresentava valores da 25OHD sérica menores que $20 \mathrm{ng} / \mathrm{mL}$. Portanto, possivelmente, os níveis séricos da $25 \mathrm{OHD}$ apenas afetam a PA quando seus valores são baixos.

Essa mesma explicação pode ser considerada no presente estudo, já que nenhum adolescente apresentou deficiência de vitamina $\mathrm{D}(<10 \mathrm{ng} / \mathrm{mL})$, e apenas $9,4 \%$ dos adolescentes apresentaram valores abaixo de $20 \mathrm{ng} / \mathrm{mL}$. Além disso, todos os adolescentes também apresentaram valores séricos de PTHi dentro da normalidade e, possivelmente, a associação do PTHi com a PA só ocorra quando seus valores se apresentam elevados, já que no estudo de Snijder e cols. (43) apenas os níveis mais altos de PTH apresentaram associação com alta prevalência de hipertensão.

No presente estudo, foi observado que, aproximadamente, $62 \%$ dos adolescentes apresentaram insuficiência de vitamina D. Resultado similar é encontrado em trabalhos de diferentes partes do mundo (44-48). No Brasil, ainda não há estudos avaliando vitamina $\mathrm{D}$ sérica em adolescentes, entretanto, Maeda e cols. (49) verificaram que, quando considerados os níveis séricos de 25OHD em adultos jovens moradores da cidade de São Paulo, aproximadamente $50 \%$ da amostra apresentava valores de $25 \mathrm{OHD}$ abaixo de $75 \mathrm{nmol} / \mathrm{L}$. 
No que se refere às limitações do estudo, a medida de PA foi realizada em uma única visita, portanto, não se pode utilizá-la para caracterizar hipertensão arterial. Essa medida deve ser usada como indicador de risco de hipertensão arterial. Deve-se considerar, também, que a amostra estudada, em sua maioria, é considerada saudável, com reduzida frequência de obesidade.

Como conclusão, os resultados do presente estudo demonstraram não haver relação entre os níveis séricos da vitamina $\mathrm{D}$ e a $\mathrm{PA}$ em adolescentes saudáveis. Apesar disso, a gordura visceral e o IMC apresentaram risco potencial para elevação da PA em adolescentes de ambos os sexos. Embora os níveis de atividade física, os hábitos alimentares e o consumo de bebidas alcoólicas não tenham se associado diretamente à $\mathrm{PA}$, a exposição contínua a esses comportamentos, ao longo da vida, pode contribuir para elevação pressórica. Considerando a importância da adolescência para a prevenção de doenças crônicas não transmissíveis, estratégias para adoção de hábitos saudáveis devem ser consideradas.

Agradecimentos: este estudo obteve auxílio financeiro da Fundação de Amparo à Pesquisa do Estado de São Paulo (Fapesp) (protocolos $n^{\circ}$ s 04/15312-9 e 05/50089-1).

Declaração: os autores declaram não haver conflitos de interesse científico neste artigo.

\section{REFERÊNCIAS}

1. Holick MF. Sunlight and vitamin D for bone health and prevention of autoimmune diseases, cancers, and cardiovascular disease. Am J Clin Nutr. 2004;80 Suppl 6:1678S-88.

2. Heaney RP. Long-latency deficiency disease: insights from calcium and vitamin D. Am J Clin Nutr. 2003;78:912-9.

3. Zittermann $A$. Vitamin $D$ in preventive medicine: are we ignoring the evidence? Br J Nutr. 2003;89:552-72.

4. Martini LA, Woods RJ. Vitamin D - Blood pressure connection: update on epidemiologic, clinical and mechanistic evidence. Nutr Rev. 2008;6:291-7.

5. Burgess ED, Hawkins RG, Watanabe M. Interaction of 1,25dihydroxyvitamin $\mathrm{D}$ and plasma renin activity in high renin essential hypertension. Am J Hypertens. 1990;3:903-5.

6. LiYC. Vitamin D regulation of the renin-angiotensin system. J Cell Biochem. 2003;88:327-31.

7. Qiao G, Kong J, Uskokovic M, Li YC. Analogs of 1alpha,25dihydroxyvitamin $\mathrm{D}(3)$ as novel inhibitors of renin biosynthesis. J Steroid Biochem Mol Biol. 2005;96:59-66.

8. Sigmund $C D$. Regulation of renin expression and blood pressure by vitamin D(3). J Clin Invest. 2002;110:155-6.

9. LiYC, Kong J, Wei M, Chen ZF, Liu SQ, Cao LP. 1,25-Dihydroxyvita$\min D(3)$ is a negative endocrine regulator of the reninangiotensin system. J Clin Invest. 2002;110:229-38.

10. Scragg R, Sowers M, Bell C. Serum 25-hydroxyvitamin D, ethnicity, and blood pressure in the Third National Health and Nutrition Examination Survey. Am J Hypertens. 2007;20:713-9.
11. Martins D, Wolf M, Pan D, Zadshir A, Tareen N, Thadhani R, et al. Prevalence of cardiovascular risk factors and the serum levels of 25-hydroxyvitamin D in the United States: data from theThird National Health and Nutrition Examination Survey. Arch Intern Med. 2007;167:1159-65.

12. Forman JP, Giovannucci E, Holmes MD, Bischoff-Ferrari HA, Tworoger SS, Willett WC, et al. Plasma 25-hydroxyvitamin D levels and risk of incident hypertension. Hypertension. 2007;49:1063-9.

13. Parikh SJ, Edelman M, Uwaifo GI, Freedman RJ, Semega-Janneh $M$, Reynolds J, et al. The relationship between obesity and serum 1,25-dihydroxy vitamin D concentrations in healthy adults. J Clin Endocrinol. 2005;89:1196-9.

14. Syme C, Abrahamowicz M, Leonard GT, Perron M, Pitiot A, Qiu X, et al. Intra-abdominal adiposity and individual components of the metabolic syndrome in adolescence: sex differences and underlying mechanisms. Arch Pediatr Adolesc Med. 2008;162:453-61.

15. Looker, AC. Body fat and vitamin D status in black versus white women. J Clin Endocrinol Metabolism. 2005;90:635-40.

16. Arunabh S, Pollack S, Yeh J, Aloia JF. Body fat content and 25hydroxyvitamin D levels in healthy women. J Clin Endocrinol Metabolism. 2003;88:157-61.

17. Center of Disease Control and Prevention. National Center for Health Statistics. CDC Growth Charts 2000. United States. Disponível em: http://www.cdc.gov/growthcharts.

18. Rodriguez G, Moreno LA, Blay MG, Blay VA, Garagorri JM, Sarría $A$, et al. Body composition in adolescents: measuraments and metabolic aspects. Int J Obes Metab Disord. 2004;28 Suppl 3:S54-8.

19. Sociedade Brasileira de Cardiologia, Sociedade Brasileira de $\mathrm{Hi}-$ pertensão, Sociedade Brasileira de Nefrologia. V Diretrizes Brasileiras de Hipertensão Arterial. São Paulo; 2006.

20. Florindo AA, Romero A, Peres SV, Silva MV, Slater B. Development and validation of a physical activity assessment questionnaire for adolescents. Rev Saude Publica. 2006;40:802-9.

21. Pate RR, Freedson PS, Sallis JF, Taylor WC, Sirard J, Trost SG, et al. Compliance with physical activity guidelines: prevalence in a population of children and youth. Ann Epidemiol. 2002;12:303-8.

22. Bischoff-Ferrari HA, Giovannucci E, Willett WC, Dietrich T, Dawson-Hughes B. Estimation of optimal serum concentrations of 25hydroxyvitamin D for multiple health outcomes. Am J Clin Nutr. 2006;84:18-28.

23. Willett W, Stampfer M. Implications of total energy intake for epidemiological analyses. In: Willett W. Nutritional epidemiology. 2. ed. New York: Oxford University Press; 1998.

24. III Consenso Brasileiro de Hipertensão Arterial. Rev Bras Clin Terap. 1998;24:231-72.

25. Silva KS, Farias Júnior JC. Fatores de risco associados à pressão arterial elevada em adolescentes. Rev Bras Med Esporte. 2007;13:237-40.

26. Pileggi C, Carbone V, Nobille CGA, Pavia M. Blood pressure and related cardiovascular disease risk factors in 6-18-year-old students in Italy. J Paediatr Child Health. 2005;41:347-52.

27. Rabbia F, Veglio F, Pinna G, Oliva S, Surgo V, Rolando B, et al. Cardiovascular risk factors in adolescents: prevalence and familial aggregation. Prev Med. 1994;23:809-15.

28. Paulus D, Saint-Remy A, Jeanjean M. Blood pressure during adolescence: a study among Belgian adolescents selected from a high cardiovascular risk population. Eur J Epidemiol. 1999;15:783-90.

29. Rosa MLG, Fonseca VM, Oigman G, Mesquita ET. Pré-hipertensão arterial e pressão de pulso aumentada em adolescentes: prevalência e fatores associados. Arq Bras Cardiol. 2006;87:46-53.

30. Monego ET, Jardim PCBV. Determinantes de risco para doenças cardiovasculares em escolares. Arq Bras Cardiol. 2006;87:37-45.

31. Guedes DP, Guedes JERP, Barbosa DS, Oliveira JA, Stanganelli LCR. Fatores de risco cardiovasculares em adolescentes: 
indicadores biológicos e comportamentais. Arq Bras Cardiol. 2006;86:439-50.

32. Ribeiro RQC, Lotufo PA, Lamounier JA, Oliveira RG, Soares JF, Botter DA. Fatores adicionais de risco cardiovascular associados ao excesso de peso em crianças e adolescentes. 0 estudo do coração de Belo Horizonte. Arq Bras Cardiol. 2006;86:408-18.

33. Nielsen GA, Andersen LB. The association between high blood pressure, physical fitness and body mass index in adolescents. Prev Med. 2003;36:229-34.

34. Weiss R, Dziura J, Burgert TS, Tamborlane W, Taksali SE, Yeckel $\mathrm{CW}$, et al. Obesity and the metabolic syndrome in children and adolescents. N Engl J Med. 2004;350:2362-74.

35. Lenchik L, RegisterTC, Hsu FC, Lohman K, Nicklas BJ, Freedman $\mathrm{Bl}$, et al. Adiponectin as a novel determinant of bone mineral density and visceral fat. Bone. 2003;33:646-51.

36. Barber TM, Hazell M, Christodoulides C, Golding SJ, Alvey C, Burling $K$, et al. Serum levels of retinol-binding protein 4 and adiponectin in women with polycystic ovary syndrome: associations with visceral fat but no evidence for fat mass-independent effects on pathogenesis in this condition. J Clin Endocrinol Metab. 2008;93:2859-65.

37. You T, Nicklas BJ, Ding J, Penninx BW, Goodpaster BH, Bauer DC, et al. The metabolic syndrome is associated with circulating adipokines in older adults across a wide range of adiposity. J Gerontol A Biol Sci Med Sci. 2008;63:414-9.

38. Hooper L, Bartlett C, Davey Smith G, Ebrahim S. Reduced dietary salt for prevention of cardiovascular disease. Cochrane Database Syst Rev. 2003;(3):CD003656.

39. Whelton PK, Appel LJ, Espeland MA, Applegate WB, Ettinger WH $\mathrm{Jr}$, Kostis JB, et al. Sodium reduction and weight loss in the treatment of hypertension in older people: a randomized controlled trial of nonpharmacologic interventions in the elderly (TONE). TONE Collaborative Research Group. JAMA. 1998;279:839-46.
40. Sowers MR, Wallace RB, Lemke JH. The association of intakes of vitamin $D$ and calcium with blood pressure among women. Am J Clin Nutr. 1985;42:135-42.

41. Pfeifer M, Begerow B, Minne HW, Nachtigall D, Hansen C. Effects of a short-term vitamin $\mathrm{D}(3)$ and calcium supplementation on blood pressure and parathyroid hormone levels in elderly women. J Clin Endocrinol Metab. 2001;86:1633-7.

42. Holick MF.The vitamin D epidemic and its health consequences. J Nutr. 2005;135:2739S-48.

43. Snijder MB, van Dam RM, Visser M, Deeg DJ, Dekker JM, Bouter $\mathrm{LM}$, et al. Adiposity in relation to vitamin $\mathrm{D}$ status and parathyroid hormone levels: a population-based study in older men and women. J Clin Endocrinol Metab. 2005;90:4119-23.

44. El-Hajj Fuleihan G, Nabulsi M, Choucair M, Salamoun M, Hajj Shahine C, Kizirian A, et al. Hypovitaminosis D in healthy schoolchildren. Pediatrics. 2001;107:E53.

45. Gordon CM, DePeter KC, Feldman HA, Grace E, Emans SJ. Prevalence of vitamin $D$ deficiency among healthy adolescents. Arch Pediatr Adolesc Med. 2004;158:531-7.

46. Livesey J, Elder P, Ellis MJ, McKenzie R, Liley B, Florkowski C. Seasonal variation in vitamin $D$ levels in the Canterbury, New Zealand population in relation to available UV radiation. N Z Med J. 2007;120:U2733.

47. HillTR, Cotter AA, Mitchell S, Boreham CA, Dubitzky W, Murray L, et al. Vitamin $D$ status and its determinants in adolescents from the Northern Ireland Young Hearts 2000 cohort. Br J Nutr. 2008;99:1061-7.

48. Mark S, Gray-Donald K, Delvin EE, O'Loughlin J, Paradis G, Levy $E$, et al. Low vitamin $D$ status in a representative sample of youth from Québec, Canada. Clin Chem. 2008;54:1283-9.

49. Maeda SS, Kunii IS, Hayashi L, Lazaretti-Castro M. The effect of sun exposure on 25-hydroxyvitamin $D$ concentrations in young healthy subjects living in the city of São Paulo, Brazil. Braz J Med Biol Res. 2007;40:1653-9. 